\title{
Letter to the Editor Regarding Strategies to Address Projected Challenges Facing Foreign Applicants in the U.S. Neurosurgery Match
}

Dear Editor,

We read with great interest the excellent original study, and the explanatory perspective, both by Chandra et al. on the 'The Path to U.S. Neurosurgical Residency for Foreign Medical Graduates: Trends from a Decade 2007-2017' and the 'Strategies to Address Projected Challenges Facing Foreign Applicants in the U.S. Neurosurgery Match' ${ }^{1,2}$ We also found valuable the correspondence by Lu on the same study. ${ }^{3}$ Taken together, they provide considerable guidance for FMGs. We add more context and discussion to these works herein.

Surgical specialties, especially neurosurgery, continue to be the most competitive residency programs for foreign medical graduates (FMGs) to get accepted to. This is evident in the US Medical Licensing Examination (USMLE) Step 1 scores of FMGs accepted to neurosurgery, which are one of the highest amongst all specialties, being a mean of 243 for the 2020 match. This is as per the third edition of the 'Charting Outcomes in the Match' report, published in July 2020 by the National Residency Matching Program (NRMP). ${ }^{4}$ The above report also indicates that amongst all specialties, the mean number of research items (presentations, publications and abstracts) by FMGs was highest in those accepted to neurosurgery. The gap in these numbers was also the largest amongst all specialities between those who matched (60.8 research items) and who did not (38.3 research items). ${ }^{4}$

FMGs may also find it useful to look at the NRMP survey of PDs. As per the last available report of 2018 , the most important factors for providing residency interviews for the 28 neurosurgery 
PDs who responded were USMLE scores (and any failed attempts), letters of recommendation (LORs) in neurosurgery, 'evidence of professionalism and ethics', audition electives, and 'demonstrated involvement in research'. ${ }^{5}$ More importantly, the three most highly cited criteria for ranking interviewed applicants were 'interaction with faculty', 'interpersonal skills', and 'interaction with housestaff'.

Chandra and colleagues' objective determination of FMG profile succeeding in the neurosurgery match is in contrast to these survey studies, which while useful, have the potential to become limited by their selection/non-response bias and existence of baseline confounders. For instance, these limitations were present in a recent work by Rajesh et al that aimed to determine value of dedicated research years after medical school for international medical graduates (IMGs). ${ }^{6}$ They reported no significant difference between match rates in surgical specialities of IMGS who did or did not take research years. They also found that just around one-fourth of general surgery (GS) program directors (PDs) that responded recommend IMGs to take research years. This is in stark contrast to Chandra et al, who objectively found that having (1) more publications, (2) higher H-index, and (3) more gap years, predicted significant match success for FMGs in neurosurgery. ${ }^{2,3}$ Similarly, Kokosis et al, while evaluating characteristics of IMGs who matched into plastic surgery residency, found that successful candidates had taken a mean of two research years. $^{7}$

We believe that the value of research, the underlying theme of the three variables above found to be important by Chandra et al, will likely increase as other objective indicators are lost. The USMLE Step 1, till now one of the most important components of neurosurgery residency application, ${ }^{1,4,5}$ has been announced to become a pass/fail examination in $2022 .{ }^{8}$ A landmark survey of over 2000 PDs reported that only $15.3 \%$ agreed with this change while over three- 
fourth of them believed this would make an objective comparison harder. ${ }^{9}$ More specifically, Huq et al. in a recent survey of approximately half of the total PDs in neurosurgery residencies found that $77 \%$ had 'always' screened candidates using Step 1 scores while around $79 \%$ differed with this change.

Finally, we recommend that IMGs, when planning for a neurosurgery residency application strategy, utilize the NRMP's 'Interactive Charting Outcomes in the Match' tool, in addition to advice from mentors and findings from the neurosurgical literature. ${ }^{11}$ It uses an exhaustive database, has much more granular data; and allows for subgroup analyses to remove the effect of confounding variables. It indicates that those non-US-IMGs, who possessed scores of 250 or above on both Step 1 and Step 2 CK, have historically had a $42 \%$ chance of matching into neurosurgery.

To conclude, findings should assure FMGs that, despite the multifactorial and complex nature of matching into neurosurgery, long-term hard work and perseverance do get rewarded. ${ }^{8,11}$

\section{References:}

1. Chandra A, Brandel MG, Wadhwa H, et al. The Path to U.S. Neurosurgical Residency for Foreign Medical Graduates: Trends from a Decade 2007-2017. World Neurosurg. 2020;137:e584-e596. doi:10.1016/j.wneu.2020.02.069

2. Chandra A, Aghi MK. Strategies to Address Projected Challenges Facing Foreign Applicants in the U.S. Neurosurgery Match. World Neurosurg. 2020;138:553-555. doi:10.1016/j.wneu.2020.03.180

3. Lu VM. Letter to the Editor Regarding "The Path to U.S. Neurosurgical Residency for Foreign Medical Graduates: Trends from a Decade 2007-2017". World Neurosurg. 2020;138:593. doi:10.1016/j.wneu.2020.03.120 
4. Charting Outcomes in the Match: International Medical Graduates, $3^{\text {rd }}$ Edition, National Resident Matching Program (NRMP) website. Published 2020 Jul. Accessed 2020 Aug 2. Available at: https://www.nrmp.org/main-residency-match-data/

5. Results of the 2018 NRMP Program Director Survey, National Resident Matching Program (NRMP) website. Published 2018 Jun. Accessed 2020 Aug 2. Available at: https://www.nrmp.org/main-residency-match-data/

6. Rajesh A, Asaad M, AlJamal YN, Enger TM, Farley DR. Value of Research Years for International Medical Graduates Applying to General Surgery Residency [published online ahead of print, 2020 Jul 31]. J Surg Educ. 2020;S1931-7204(20)30123-9.

7. Kokosis G, Leto Barone AA, Grzelak MJ, et al. International Medical Graduates in the US Plastic Surgery Residency: Characteristics of Successful Applicants. Eplasty. 2018;18:e33. Published 2018 Nov 27.

8. Boulet JR, Pinsky WW. Reporting a Pass/Fail Outcome for USMLE Step 1. [published online ahead of print, June 2, 2020]. Acad Med. doi:10.1097/ACM.0000000000003534

9. Makhoul AT, Pontell ME, Ganesh Kumar N, Drolet BC. Objective Measures Needed Program Directors' Perspectives on a Pass/Fail USMLE Step 1. N Engl J Med. 2020;382(25):2389-2392

10. Huq S, Khalafallah AM, Botros D, et al. Perceived impact of USMLE Step 1 pass/fail scoring change on neurosurgery: program director survey. [published online ahead of print June 19 2020] J Neurosurg. doi:10.3171/2020.4.jns20748

11. Interactive Charting Outcomes in the match, NRMP website. Accessed 2020 Aug 2. Available at: https://www.nrmp.org/interactive-charting-outcomes-in-the-match/ 